\title{
Existence of positive solutions of advanced differential equations
}

Qiaoluan Li, Xiaojing Liu, Feifei Cui and Weina Li

${ }^{*}$ Correspondence: ql171125@163.com

College of Mathematics and Information Science, Hebei Normal University, Shijiazhuang, 050024, P.R. China

\section{Abstract}

In this paper, we study the advanced differential equations

$$
\left[r(t)\left|x^{\prime}(t)\right|^{\alpha-1} x^{\prime}(t)\right]^{\prime}+\sum_{i=1}^{n} p_{i}(t)\left|x\left(t+\tau_{i}(t)\right)\right|^{\alpha-1} x\left(t+\tau_{i}(t)\right)=0
$$

and

$$
\left[r(t)(y(t)-P(t) y(t-\tau))^{\prime}\right]^{\prime}+\sum_{i=1}^{n} p_{i}(t) f(y(t+\sigma))=0 .
$$

By using the generalized Riccati transformation and the Schauder-Tyichonoff theorem, we establish the conditions for the existence of positive solutions of the above equations.

MSC: $34 \mathrm{~K} 11 ; 39 \mathrm{~A} 10$

Keywords: advanced differential equations; positive solutions; existence

\section{Introduction}

In the last years, oscillation and nonoscillation of differential equations attracted a considerable attention. Many results have been obtained, and we refer the reader to the papers [1-20].

In 2008, Luo et al. [11] investigated the existence of positive periodic solutions of the following two kinds of neutral functional differential equations:

$$
(x(t)-c x(t-\tau(t)))^{\prime}=-a(t) x(t)+f(t, x(t-\tau(t)))
$$

and

$$
\left(x(t)-c \int_{-\infty}^{0} Q(r) x(t+r) d r\right)^{\prime}=-a(t) x(t)+b(t) \int_{-\infty}^{0} Q(r) f(t, x(t+r)) d r,
$$

where $a, b \in C(R,(0, \infty)), \tau \in C(R, R), f \in C(R \times R, R)$, and $a(t), b(t), \tau(t), f(t, x)$ are $\omega$-periodic functions, $\omega>0, Q(r) \in C((-\infty, 0],[0, \infty)), \int_{-\infty}^{0} Q(r) d r=1$, and $\omega,|c|<1$ are constants.

C 2013 Li et al.; licensee Springer. This is an Open Access article distributed under the terms of the Creative Commons Attribution License (http://creativecommons.org/licenses/by/2.0), which permits unrestricted use, distribution, and reproduction in any medium, provided the original work is properly cited. 
Péics et al. [15] obtained the existence of positive solutions of half-linear delay differential equations

$$
\left[\left|x^{\prime}(t)\right|^{\alpha-1} x^{\prime}(t)\right]^{\prime}+\sum_{i=1}^{n} p_{i}(t)\left|x\left(t-\tau_{i}(t)\right)\right|^{\alpha-1} x\left(t-\tau_{i}(t)\right)=0
$$

where $t \geq t_{0}$ and $\alpha>0, \tau_{i}(t) \leq t$.

Zhang et al. [19] obtained the existence of nonoscillatory solutions of the first-order linear neutral delay differential equation

$$
[x(t)+P(t) x(t-\tau)]^{\prime}+Q_{1}(t) x\left(t-\sigma_{1}\right)-Q_{2}(t) x\left(t-\sigma_{2}\right)=0,
$$

where $P \in C\left(\left[t_{0}, \infty\right), R\right), \tau \in(0, \infty), \sigma_{1}, \sigma_{2} \in[0, \infty), Q_{1}, Q_{2}>0$.

In this paper, we consider the advanced differential equation

$$
\left[r(t)\left|x^{\prime}(t)\right|^{\alpha-1} x^{\prime}(t)\right]^{\prime}+\sum_{i=1}^{n} p_{i}(t)\left|x\left(t+\tau_{i}(t)\right)\right|^{\alpha-1} x\left(t+\tau_{i}(t)\right)=0,
$$

where $t \geq t_{0}$ and $\alpha>0$.

Throughout this work, we always assume that the following conditions hold:

$\left(\mathrm{H}_{1}\right) p_{i} \in C\left(\left[t_{0}, \infty\right), R\right), i=1,2,3, \ldots, n$;

$\left(\mathrm{H}_{2}\right) \tau_{i} \in C\left(\left[t_{0}, \infty\right), R^{+}\right), i=1,2,3, \ldots, n$, and $0<r(t) \leq k$.

For convenience, we introduce the notation

$$
\eta^{\alpha^{*}}=|\eta|^{\alpha-1} \eta, \quad \alpha>0 .
$$

It is convenient to rewrite (1.1) in the form

$$
\left[r(t)\left|x^{\prime}(t)\right|^{\alpha^{*}}\right]^{\prime}+\sum_{i=1}^{n} p_{i}(t)\left|x\left(t+\tau_{i}(t)\right)\right|^{\alpha^{*}}=0 .
$$

Definition 1.1 A function $x$ is said to be a solution of Eq. (1.1) if $x \in C^{1}([T, \infty), R), T \geq t_{0}$, which has the property $\left|x^{\prime}\right|^{\alpha-1} x^{\prime} \in C^{1}([T, \infty), R)$ and it satisfies Eq. (1.1) for $t \geq T$. We say that a solution of Eq. (1.1) is oscillatory if it has arbitrarily large zeros. Otherwise, it is nonoscillatory.

One of the most important methods of the study of nonoscillation is the method of generalized characteristic equation [6]. The method was applied to second-order halflinear equations without delay, for example, in $[8,9]$. Concerning cases with advanced, let us apply the Riccati-transformation

$$
x(t)=\exp \left(\int_{t_{0}}^{t}(\omega(s))^{\left(\frac{1}{\alpha}\right)^{n}} d s\right) .
$$

By (1.4), we have

$$
x^{\prime}(t)=\left(\exp \left(\int_{t_{0}}^{t} \omega(s)^{\left(\frac{1}{\alpha}\right)^{*}} d s\right)\right)^{\prime}=\omega(t)^{\left(\frac{1}{\alpha}\right)^{*}} \exp \left(\int_{t_{0}}^{t} \omega(s)^{\left(\frac{1}{\alpha}\right)^{*}} d s\right),
$$




$$
\left(x\left(t+\tau_{i}(t)\right)\right)^{\alpha^{*}}=\exp \left(\alpha^{*} \int_{t_{0}}^{t+\tau_{i}(t)} \omega(s)^{\left(\frac{1}{\alpha}\right)^{*}} d s\right)
$$

From (1.3), we obtain

$$
\left[r(t) \omega(t) \exp \left(\alpha^{*} \int_{t_{0}}^{t} \omega(s)^{\left(\frac{1}{\alpha}\right)^{*}} d s\right)\right]^{\prime}+\sum_{i=1}^{n} p_{i}(t) \exp \left(\alpha^{*} \int_{t_{0}}^{t+\tau_{i}(t)} \omega(s)^{\left(\frac{1}{\alpha}\right)^{*}} d s\right)=0
$$

Since

$$
\begin{aligned}
& {\left[r(t) \omega(t) \exp \left(\alpha^{*} \int_{t_{0}}^{t} \omega(s)^{\left(\frac{1}{\alpha}\right)^{*}} d s\right)\right]^{\prime}} \\
& =(r(t) \omega(t))^{\prime} \exp \left(\alpha^{*} \int_{t_{0}}^{t} \omega(s)^{\left(\frac{1}{\alpha}\right)^{*}} d s\right)+r(t) \omega(t)\left(\exp \left(\alpha^{*} \int_{t_{0}}^{t} \omega(s)^{\left(\frac{1}{\alpha}\right)^{*}} d s\right)\right)^{\prime} \\
& =\left(r^{\prime}(t) \omega(t)+r(t) \omega^{\prime}(t)\right) \exp \left(\alpha^{*} \int_{t_{0}}^{t} \omega(s)^{\left(\frac{1}{\alpha}\right)^{*}} d s\right)+\alpha^{* \prime \prime} r(t)|\omega(t)|^{1+\frac{1}{\alpha}} \\
& \quad \times \exp \left(\alpha^{*} \int_{t_{0}}^{t} \omega(s)^{\left(\frac{1}{\alpha}\right)^{*}} d s\right),
\end{aligned}
$$

it is convenient to rewrite (1.5) in the form

$$
r^{\prime}(t) \omega(t)+r(t) \omega^{\prime}(t)+\alpha r(t)|\omega(t)|^{1+\frac{1}{\alpha}}+\sum_{i=1}^{n} p_{i}(t) \exp \left(\alpha \int_{t}^{t+\tau_{i}(t)} \omega(s)^{\left(\frac{1}{\alpha}\right)^{\circ}} d s\right)=0
$$

\section{Preliminaries}

Lemma 2.1 Suppose that $\left(\mathrm{H}_{1}\right)$ and $\left(\mathrm{H}_{2}\right)$ hold. Then the following statements are equivalent:

(i) Eq. (1.1) has an eventually positive solution;

(ii) There is a function $\omega \in C^{1}([T, \infty), R), T \geq t_{0}$, such that $\omega$ solves the Riccati equation (1.6).

Proof (i) $\Rightarrow$ (ii). Let $x$ be an eventually positive solution of Eq. (1.1) such that $x(t)>0$ for $t \geq T \geq t_{0}$. The function $\omega$ defined by

$$
\omega(t)=\left(\frac{x^{\prime}(t)}{x(t)}\right)^{\alpha^{\prime \prime}}, \quad t \geq T
$$

is continuous.

We will show that it is a solution of $(1.6)$ on $[T, \infty)$. By $(1.2)$ and observing that

$$
\begin{aligned}
& \omega(t)=\left(\frac{x^{\prime}(t)}{x(t)}\right)^{\alpha^{\prime \prime}}=\left|\frac{x^{\prime}(t)}{x(t)}\right|^{\alpha-1} \frac{x^{\prime}(t)}{x(t)}, \\
& \frac{x^{\prime}(t)}{x(t)}=|\omega(t)|^{\frac{1}{\alpha}-1} \omega(t)=\omega(t)^{\left(\frac{1}{\alpha}\right)^{\prime}},
\end{aligned}
$$

it follows that

$$
x(t)=x(T) \exp \left(\int_{T}^{t} \omega(s)^{\left(\frac{1}{\alpha}\right)^{\prime \prime}} d s\right) .
$$


Dividing both sides of (1.1) by $|x(t)|^{\alpha-1} x(t)$ gives that

$$
\frac{\left[r(t)\left|x^{\prime}(t)\right|^{\alpha-1} x^{\prime}(t)\right]^{\prime}}{|x(t)|^{\alpha-1} x(t)}+\sum_{i=1}^{n} p_{i}(t) \frac{\left|x\left(t+\tau_{i}(t)\right)\right|^{\alpha-1} x\left(t+\tau_{i}(t)\right)}{|x(t)|^{\alpha-1} x(t)}=0 .
$$

From the definition of $\omega$, we obtain

$$
\left|x^{\prime}(t)\right|^{\alpha-1} x^{\prime}(t)=\omega(t)|x(t)|^{\alpha-1} x(t)=\omega(t) x^{\alpha}(t) .
$$

Further

$$
\begin{aligned}
\left(r(t)\left|x^{\prime}(t)\right|^{\alpha-1} x^{\prime}(t)\right)^{\prime} & =\left(r(t) \omega(t) x^{\alpha}(t)\right)^{\prime} \\
& =r^{\prime}(t) \omega(t) x^{\alpha}(t)+r(t) \omega^{\prime}(t) x^{\alpha}(t)+\alpha r(t) \omega(t) x^{\alpha-1}(t) x^{\prime}(t)
\end{aligned}
$$

and

$$
\begin{aligned}
& \frac{x\left(t+\tau_{i}(t)\right)}{x(t)}=\exp \left(\int_{t}^{t+\tau_{i}(t)} \omega(s)^{\left(\frac{1}{\alpha}\right)^{*}} d s\right)>0, \\
& \left|\frac{x\left(t+\tau_{i}(t)\right)}{x(t)}\right|^{\alpha^{*}}=\exp \left(\alpha \int_{t}^{t+\tau_{i}(t)} \omega(s)^{\left(\frac{1}{\alpha}\right)^{*}} d s\right) .
\end{aligned}
$$

By substituting (2.2), (2.3) into (2.1), we get

$$
\begin{aligned}
& \frac{\left[r(t)\left|x^{\prime}(t)\right|^{\alpha-1} x^{\prime}(t)\right]^{\prime}}{|x(t)|^{\alpha-1} x(t)}+\sum_{i=1}^{n} p_{i}(t) \frac{\left|x\left(t+\tau_{i}(t)\right)\right|^{\alpha-1} x\left(t+\tau_{i}(t)\right)}{|x(t)|^{\alpha-1} x(t)} \\
& =\frac{r^{\prime}(t) \omega(t) x^{\alpha}(t)+r(t) \omega^{\prime}(t) x^{\alpha}(t)+\alpha r(t) \omega(t) x^{\alpha-1}(t) x^{\prime}(t)}{x^{\alpha}(t)} \\
& \quad+\sum_{i=1}^{n} p_{i}(t) \exp \left(\alpha \int_{t}^{t+\tau_{i}(t)} \omega(s)^{\left(\frac{1}{\alpha}\right)^{*}} d s\right) \\
& =0 .
\end{aligned}
$$

We obtain (1.6), and the proof of (i) $\Rightarrow$ (ii) is complete.

(ii) $\Rightarrow$ (i). Let $\omega$ be a continuously differentiable solution of Eq. (1.6) for $t \geq T \geq t_{0}$. We show that a function $x$ defined by

$$
x(t)=\exp \left(\int_{T}^{t} \omega(s)^{\left(\frac{1}{\alpha}\right)^{*}} d s\right)
$$

is the solution of Eq. (1.1).

Since

$$
\begin{aligned}
& \frac{x^{\prime}(t)}{x(t)}=\omega(t)^{\left(\frac{1}{\alpha}\right)^{*},} \\
& \left(x^{\prime}(t)\right)^{\alpha^{*}}=(x(t))^{\alpha^{*}} \omega(t)=x^{\alpha}(t) \omega(t) .
\end{aligned}
$$


By (1.6), we obtain

$$
\begin{aligned}
{\left[r(t)\left(x^{\prime}(t)\right)^{\alpha^{\prime \prime}}\right]^{\prime} } & =\left(r(t) \omega(t) x^{\alpha}(t)\right)^{\prime} \\
& =r^{\prime}(t) \omega(t) x^{\alpha}(t)+r(t) \omega^{\prime}(t) x^{\alpha}(t)+\alpha r(t) \omega(t) x^{\alpha-1}(t) x^{\prime}(t) \\
& =r^{\prime}(t) \omega(t) x^{\alpha}(t)+r(t) \omega^{\prime}(t) x^{\alpha}(t)+\alpha r(t) x^{\alpha}(t)|\omega(t)|^{1+\frac{1}{\alpha}} \\
& =-\sum_{i=1}^{n} p_{i}(t) \exp \left(\alpha \int_{t}^{t+\tau_{i}(t)} \omega(s)^{\left(\frac{1}{\alpha}\right)^{*}} d s\right) \exp \left(\alpha \int_{T}^{t} \omega(s)^{\left(\frac{1}{\alpha}\right)^{*}} d s\right) \\
& =-\sum_{i=1}^{n} p_{i}(t) \exp \left(\alpha \int_{T}^{t+\tau_{i}(t)} \omega(s)^{\left(\frac{1}{\alpha}\right)^{*}} d s\right) \\
& =-\sum_{i=1}^{n} p_{i}(t) x^{\alpha}\left(t+\tau_{i}(t)\right),
\end{aligned}
$$

thus,

$$
\left[r(t)\left(x^{\prime}(t)\right)^{\alpha^{\prime \prime}}\right]^{\prime}+\sum_{i=1}^{n} p_{i}(t) x^{\alpha}\left(t+\tau_{i}(t)\right)=0, \quad t \geq T .
$$

The proof of (ii) $\Rightarrow$ (i) is complete. The proof is complete.

Lemma 2.2 Suppose that $\left(\mathrm{H}_{1}\right)$ and $\left(\mathrm{H}_{2}\right)$ hold. The following statements are equivalent:

(a) There is a solution $\omega \in C^{1}([T, \infty), R)$ of the Riccati equation (1.6) for some $T \geq t_{0}$ such that

$$
\int_{t}^{\infty}\left[\sum_{i=1}^{n} p_{i}(s) \exp \left(\alpha \int_{s}^{s+\tau_{i}(s)} \omega(\xi)^{\left(\frac{1}{\alpha}\right)^{*}} d \xi\right)\right] d s<\infty
$$

(b) There is a function $u \in C([T, \infty), R)$ for some $T \geq t_{0}$ such that

$$
\begin{aligned}
u(t)= & \frac{1}{r(t)}\left\{\alpha \int_{t}^{\infty} r(s)|u(s)|^{1+\frac{1}{\alpha}} d s\right. \\
& \left.+\int_{t}^{\infty}\left[\sum_{i=1}^{n} p_{i}(s) \exp \left(\alpha \int_{s}^{s+\tau_{i}(s)} \omega(\xi)^{\left(\frac{1}{\alpha}\right)^{*}} d \xi\right)\right] d s\right\} .
\end{aligned}
$$

Proof (a) $\Rightarrow$ (b). Let $\omega=u$ be a solution of Eq. (1.6) for $t \geq T \geq t_{0}$ and with the property (2.4). Let $t_{1} \geq t \geq T$ be fixed arbitrarily and integrate (1.6) over $\left[t, t_{1}\right]$ :

$$
\begin{aligned}
u\left(t_{1}\right) r\left(t_{1}\right)-u(t) r(t)= & -\alpha \int_{t}^{t_{1}} r(s)|u(s)|^{1+\frac{1}{\alpha}} d s \\
& -\int_{t}^{t_{1}}\left[\sum_{i=1}^{n} p_{i}(s) \exp \left(\alpha \int_{s}^{s+\tau_{i}(s)}|u(\xi)|^{\frac{1}{\alpha}-1} u(\xi) d \xi\right)\right] d s .
\end{aligned}
$$

We claim that

$$
\int_{t}^{\infty} r(s)|u(s)|^{1+\frac{1}{\alpha}} d s<\infty
$$


Assuming the contrary, if $\int_{t}^{\infty} r(s)|u(s)|^{1+\frac{1}{\alpha}} d s=\infty$, then in view of (2.6) there is $T_{1} \geq t$ such that

$$
\begin{aligned}
& u\left(t_{1}\right) r\left(t_{1}\right)+\alpha \int_{T_{1}}^{t_{1}} r(s)|u(s)|^{1+\frac{1}{\alpha}} d s \\
&= u(t) r(t)-\alpha \int_{t}^{T_{1}} r(s)|u(s)|^{1+\frac{1}{\alpha}} d s \\
&-\int_{t}^{t_{1}}\left[\sum_{i=1}^{n} p_{i}(s) \exp \left(\alpha \int_{s}^{s+\tau_{i}(s)}|u(\xi)|^{\frac{1}{\alpha}-1} u(\xi) d \xi\right)\right] d s \\
& \leq-1
\end{aligned}
$$

for $t_{1} \geq T_{1} \geq t$, or equivalently,

$$
-u\left(t_{1}\right) r\left(t_{1}\right) \geq 1+\alpha \int_{T_{1}}^{t_{1}} r(s)|u(s)|^{1+\frac{1}{\alpha}} d s, \quad t_{1} \geq T_{1} .
$$

Then we have

$$
u\left(t_{1}\right) \leq 0 .
$$

From $u(t)=\left(\frac{x^{\prime}(t)}{x(t)}\right)^{\alpha^{*}}$, it follows that $x^{\prime}\left(t_{1}\right)<0, t_{1} \geq T_{1}$. Dividing both sides of (2.8) by $1+$ $\alpha \int_{T_{1}}^{t_{1}} r(s)|u(s)|^{1+\frac{1}{\alpha}} d s>0$ gives that

$$
\frac{\left|u\left(t_{1}\right)\right|^{1+\frac{1}{\alpha}} r\left(t_{1}\right)}{1+\alpha \int_{T_{1}}^{t_{1}} r(s)|u(s)|^{1+\frac{1}{\alpha}} d s} \geq\left(-u\left(t_{1}\right)\right)^{\frac{1}{\alpha}}=-\frac{x^{\prime}\left(t_{1}\right)}{x\left(t_{1}\right)}, \quad t_{1} \geq T_{1} .
$$

Integrating the above inequality over $\left[T_{1}, t_{1}\right]$ then yields

$$
\frac{1}{\alpha} \ln \left(1+\alpha \int_{T_{1}}^{t_{1}} r(s)|u(s)|^{1+\frac{1}{\alpha}} d s\right) \geq \ln \left(\frac{x\left(T_{1}\right)}{x\left(t_{1}\right)}\right) .
$$

Combining with (2.8), we have

$$
\left(-r\left(t_{1}\right) u\left(t_{1}\right)\right)^{\frac{1}{\alpha}} \geq \frac{x\left(T_{1}\right)}{x\left(t_{1}\right)}, \quad t_{1} \geq T_{1}
$$

and

$$
-r^{\frac{1}{\alpha}}\left(t_{1}\right) x^{\prime}\left(t_{1}\right) \geq x\left(T_{1}\right)
$$

Integrating the last inequality and using $0<r(t) \leq k$, we see that $\lim _{t \rightarrow \infty} x(t)=-\infty$, which contradicts the assumption that $x(t)$ is eventually positive. Therefore (2.7) must hold.

Let $t_{1} \rightarrow \infty$ in (2.6). Using (2.4) and (2.7), we get $\lim _{t_{1} \rightarrow \infty} r\left(t_{1}\right) u\left(t_{1}\right)=0$. So,

$$
u(t)=\frac{1}{r(t)}\left\{\alpha \int_{t}^{\infty} r(s)|u(s)|^{1+\frac{1}{\alpha}} d s+\int_{t}^{\infty}\left[\sum_{i=1}^{n} p_{i}(s) \exp \left(\alpha \int_{s}^{s+\tau_{i}(s)} u(\xi)^{\left(\frac{1}{\alpha}\right)^{*}} d \xi\right)\right] d s\right\}
$$

must hold. 
(b) $\Rightarrow$ (a). Assume that there is a function $u(t)$ satisfying Eq. $(2.5)$ on $[T, \infty)$. Differentiation of (2.5) then shows that $u=\omega$ is a solution of (1.6) for $t \geq T$, and it satisfies (2.4). The proof of $(b) \Rightarrow(a)$ is complete.

\section{Main results}

Theorem 3.1 Assume that there exist $T \geq t_{0}$ and functions $\beta, \gamma \in C([T, \infty), R)$ such that $\beta(t) \leq \gamma(t)$

$$
\begin{aligned}
& \int_{t}^{\infty}\left[\sum_{i=1}^{n}\left|p_{i}(s)\right| \exp \left(\alpha \int_{s}^{s+\tau_{i}(s)} \gamma(\xi)^{\left(\frac{1}{\alpha}\right)^{*}} d \xi\right)\right] d s<\infty \\
& \beta(t) \leq v(t) \leq \gamma(t) \text { implies that } S v \text { is defined and } \beta(t) \leq(S \nu)(t) \leq \gamma(t)
\end{aligned}
$$

for every function $v \in C([T, \infty), R)$, where

$$
\begin{aligned}
(S \nu)(t)= & \frac{1}{r(t)}\left\{\alpha \int_{t}^{\infty} r(s)|v(s)|^{1+\frac{1}{\alpha}} d s\right. \\
& \left.+\int_{t}^{\infty}\left[\sum_{i=1}^{n} p_{i}(s) \exp \left(\alpha \int_{s}^{s+\tau_{i}(s)} v(\xi)^{\left(\frac{1}{\alpha}\right)^{*}} d \xi\right)\right] d s\right\} .
\end{aligned}
$$

Then there exists a continuous solution $u(t)$ of Eq. (2.5) which satisfies the inequality $\beta(t) \leq$ $u(t) \leq \gamma(t)$.

Proof Let $T_{1}$ and $T_{2}$ be real numbers such that $T \leq T_{1} \leq T_{2}<\infty$. Then $\left[T_{1}, T_{2}\right]$ is an arbitrary compact subinterval of $[T, \infty)$ and set

$$
\begin{aligned}
& L=\max _{T_{1} \leq t \leq T_{2}}\{\max \{|\beta(t)|,|\gamma(t)|\}\}, \quad \tau=\max _{T_{1} \leq t \leq T_{2}}\left\{\max _{1 \leq i \leq n} \tau_{i}(t)\right\} \\
& L_{1}=L^{\frac{1}{\alpha}-1} e^{\alpha \tau L^{\frac{1}{\alpha}}}, \quad N=\min _{T_{1} \leq t \leq T_{2}} r(t), \\
& M=\max _{T_{1} \leq t \leq T_{2}} \sum_{i=1}^{n}\left|p_{i}(t)\right|, \quad c=\frac{k(\alpha+1) L^{\frac{1}{\alpha}}+M L_{1} \tau}{N} .
\end{aligned}
$$

Define

$$
F=\{v \in C([T, \infty), R) \mid \beta(t) \leq v(t) \leq \gamma(t), t \in[T, \infty)\}
$$

It follows from (3.1) and (3.2), that the operator $S$ is defined for $v \in F$ and satisfies

$$
\int_{t}^{\infty} r(\zeta)|v(\zeta)|^{1+\frac{1}{\alpha}} d \zeta<\infty
$$

By (3.2), we see that the functions in the image set $S F$ are uniformly bounded on any finite interval of $[T, \infty)$.

To prove that the functions in $S F$ are equicontinuous on any finite interval of $[T, \infty)$, we choose the finite interval $\left[T_{1}, T_{2}\right]$ as before, and let $t_{1}$ and $t_{2}$ be two arbitrary numbers 
from $\left[T_{1}, T_{2}\right]$. Since $\frac{1}{r(t)}$ is continuous on $\left[T_{1}, T_{2}\right], \forall \varepsilon>0, \exists \delta_{1}>0$, such that for $\left|t_{1}-t_{2}\right|<\delta_{1}$, we have

$$
\left|\frac{1}{r\left(t_{1}\right)}-\frac{1}{r\left(t_{2}\right)}\right|<\frac{\varepsilon}{2} \Rightarrow \frac{1}{r\left(t_{1}\right)}<\frac{1}{r\left(t_{2}\right)}+\frac{\varepsilon}{2} .
$$

Further,

$$
\begin{aligned}
\mid S v & \left.t_{1}\right)-S v\left(t_{2}\right) \mid \\
= & \frac{1}{r\left(t_{1}\right)}\left\{\alpha \int_{t_{1}}^{\infty} r(s)|v(s)|^{1+\frac{1}{\alpha}} d s+\int_{t_{1}}^{\infty}\left[\sum_{i=1}^{n} p_{i}(s) \exp \left(\alpha \int_{s}^{s+\tau_{i}(s)} v(\xi)^{\left(\frac{1}{\alpha}\right)^{*}} d \xi\right)\right] d s\right\} \\
& -\frac{1}{r\left(t_{2}\right)}\left\{\alpha \int_{t_{2}}^{\infty} r(s)|v(s)|^{1+\frac{1}{\alpha}} d s\right. \\
& \left.+\int_{t_{2}}^{\infty}\left[\sum_{i=1}^{n} p_{i}(s) \exp \left(\alpha \int_{s}^{s+\tau_{i}(s)} v(\xi)^{\left(\frac{1}{\alpha}\right)^{\infty}} d \xi\right)\right] d s\right\} \mid \\
\leq & \frac{1}{r\left(t_{2}\right)}\left\{\alpha \int_{t_{1}}^{t_{2}} r(s)|v(s)|^{1+\frac{1}{\alpha}} d s+\int_{t_{1}}^{t_{2}}\left[\sum_{i=1}^{n}\left|p_{i}(s)\right| \exp \left(\alpha \int_{s}^{s+\tau_{i}(s)} v(\xi)^{\left(\frac{1}{\alpha}\right)^{*}} d \xi\right)\right] d s\right\} \mid \\
& +\frac{\varepsilon}{2}\left|\left\{\alpha \int_{t_{1}}^{\infty} r(s)|v(s)|^{1+\frac{1}{\alpha}} d s+\int_{t_{1}}^{\infty}\left[\sum_{i=1}^{n}\left|p_{i}(s)\right| \exp \left(\alpha \int_{s}^{s+\tau_{i}(s)} v(\xi)^{\left(\frac{1}{\alpha}\right)^{*}} d \xi\right)\right] d s\right\}\right| \\
\leq & \frac{1}{N}\left(\alpha k L^{1+\frac{1}{\alpha}}+M e^{\alpha \tau L}\right) \mid \\
& +\frac{\varepsilon}{2}\left|\left\{\alpha \int_{t_{1}}^{\infty} r(s)|v(s)|^{1+\frac{1}{\alpha}} d s+\int_{t_{1}}^{\infty}\left[\sum_{i=1}^{n}\left|p_{i}(s)\right| \exp \left(\alpha \int_{s}^{s+\tau_{i}(s)} v(\xi)^{\left(\frac{1}{\alpha}\right)^{*}} d \xi\right)\right] d s\right\}\right| .
\end{aligned}
$$

Due to (3.1) and (3.4), there exists $\delta_{2}$ such that for $\left|t_{1}-t_{2}\right|<\delta_{2},\left|S v\left(t_{1}\right)-S v\left(t_{2}\right)\right|<\varepsilon$, hence $S F$ is equicontinuous.

Let the sequence $\left\{v_{n}(t)\right\} \in F$ tend to $v(t)$ uniformly on any finite interval $(n \rightarrow \infty)$. In particular, the convergence is uniform on the interval $\left[T_{1}, T_{2}\right]$. Using the mean value theorem, we have

$$
\left.|r(s)| v(s)\right|^{1+\frac{1}{\alpha}}-r(s)\left|v_{n}(s)\right|^{1+\frac{1}{\alpha}}\left|=\left(1+\frac{1}{\alpha}\right) r(s)\right| v(s)-\left.v_{n}(s)|| \sigma(s)\right|^{\frac{1}{\alpha}}
$$

where $|\sigma(s)|$ is between $|v(s)|$ and $\left|v_{n}(s)\right|$, and similarly

$$
\begin{aligned}
& \exp \left(\alpha \int_{s}^{s+\tau_{i}(s)}\left|v_{n}(\xi)\right|^{\frac{1}{\alpha}-1} v_{n}(\xi) d \xi\right)-\exp \left(\alpha \int_{s}^{s+\tau_{i}(s)}|v(\xi)|^{\frac{1}{\alpha}-1} v(\xi) d \xi\right) \\
& =\alpha e^{\sigma_{i}(s)} \int_{s}^{s+\tau_{i}(s)}\left(\left|v_{n}(\xi)\right|^{\frac{1}{\alpha}-1} v_{n}(\xi)-|v(\xi)|^{\frac{1}{\alpha}-1} v(\xi)\right) d \xi
\end{aligned}
$$

for every $i=1,2,3, \ldots, n$ and $T_{1} \leq s \leq T_{2}$, where $\sigma_{i}(s)$ is between $\alpha \int_{s}^{s+\tau_{i}(s)}|\nu(\xi)|^{\frac{1}{\alpha}-1} v(\xi) d \xi$ and $\alpha \int_{s}^{s+\tau_{i}(s)}\left|v_{n}(\xi)\right|^{\frac{1}{\alpha}-1} v_{n}(\xi) d \xi$. 
Since $\left|\sigma_{i}(s)\right| \leq \alpha \tau L^{\frac{1}{\alpha}}$ for $T_{1} \leq t \leq T_{2}$, we obtain

$$
\begin{aligned}
& \left|\exp \left(\alpha \int_{s}^{s+\tau_{i}(s)}\left|v_{n}(\xi)\right|^{\frac{1}{\alpha}-1} v_{n}(\xi) d \xi\right)-\exp \left(\alpha \int_{s}^{s+\tau_{i}(s)}|v(\xi)|^{\frac{1}{\alpha}-1} v(\xi) d \xi\right)\right| \\
& \quad \leq\left.\alpha e^{\alpha \tau L^{\frac{1}{\alpha}}} \int_{s}^{s+\tau_{i}(s)}|| v_{n}(\xi)\right|^{\frac{1}{\alpha}-1} v_{n}(\xi)-|v(\xi)|^{\frac{1}{\alpha}-1} v(\xi) \mid d \xi \\
& \quad \leq L^{\frac{1}{\alpha}-1} e^{\alpha \tau L^{\frac{1}{\alpha}}} \int_{s}^{s+\tau_{i}(s)}\left|v_{n}(\xi)-v(\xi)\right| d \xi .
\end{aligned}
$$

Hence,

$$
\begin{aligned}
& \left|S v(t)-S v_{n}(t)\right| \\
& \leq \lim _{T_{2} \rightarrow \infty} \frac{1}{r(t)}\left\{\left.\alpha \int_{t}^{T_{2}}|r(s)| v(s)\right|^{1+\frac{1}{\alpha}}-r(s)\left|v_{n}(s)\right|^{1+\frac{1}{\alpha}} \mid d s+\int_{t}^{T_{2}}\left[\sum_{i=1}^{n}\left|p_{i}(s)\right|\right.\right. \\
& \left.\left.\quad \times\left|\exp \left(\alpha \int_{s}^{s+\tau_{i}(s)}\left|v_{n}(\xi)\right|^{\frac{1}{\alpha}-1} v_{n}(\xi) d \xi\right)-\exp \left(\alpha \int_{s}^{s+\tau_{i}(s)}|v(\xi)|^{\frac{1}{\alpha}-1} v(\xi) d \xi\right)\right| d s\right]\right\} \\
& \leq \frac{1}{r(t)}\left[\lim _{T_{2} \rightarrow \infty}(\alpha+1) L^{\frac{1}{\alpha}} \int_{t}^{T_{2}} r(s)\left|v(s)-v_{n}(s)\right| d s\right. \\
& \left.\quad+M L_{1} \int_{t}^{T_{2}} \int_{s}^{s+\tau}\left|v(s)-v_{n}(s)\right| d \xi d s\right] .
\end{aligned}
$$

The uniform convergence $v_{n}(t)-v(t) \rightarrow 0$ on any finite interval of $[T, \infty)$ implies that if $n$ is sufficiently large,

$$
\left|v(t)-v_{n}(t)\right|<\delta, \quad T_{1} \leq t \leq T_{2},
$$

where $\delta=\frac{\varepsilon}{T_{2}}$, and hence we obtain

$$
\begin{aligned}
\left|S v(t)-S v_{n}(t)\right| & \leq \frac{1}{r(t)}\left[\lim _{T_{2} \rightarrow \infty}(\alpha+1) L^{\frac{1}{\alpha}} k \delta\left(T_{2}-t\right)+M L_{1} \tau \delta\left(T_{2}-t\right)\right] \\
& \leq \lim _{T_{2} \rightarrow \infty} \frac{1}{N}\left[(\alpha+1) L^{\frac{1}{\alpha}} k+M L_{1} \tau\right] \delta T_{2} \\
& \leq \lim _{T_{2} \rightarrow \infty} \frac{1}{N}\left[(\alpha+1) L^{\frac{1}{\alpha}} k+M L_{1} \tau\right] \varepsilon \\
& =c \varepsilon
\end{aligned}
$$

for $T_{1} \leq t \leq T_{2}$. Thus, $S v_{n}(t) \rightarrow S v(t)$ uniformly on a finite interval.

We obtained that the conditions of the Schauder-Tyichonoff theorem are satisfied, hence the mapping $S$ has at least one fixed point $v$ in $F$, and because $v(t)=(S v)(t)$ for $t \geq T, v$ is the continuous solution of Eq. (2.5).

Theorem 3.2 Assume that $\left(\mathrm{H}_{1}\right),\left(\mathrm{H}_{2}\right)$ hold and there exists a positive function $\mu(t)$ for $t \geq T \geq t_{0}$ such that

$$
\frac{1}{r(t)} \int_{t}^{\infty}\left[\alpha r(s) \mu^{1+\frac{1}{\alpha}}(s)+\sum_{i=1}^{n}\left|p_{i}(s)\right| \exp \left(\alpha \int_{s}^{s+\tau_{i}(s)} \mu^{\frac{1}{\alpha}}(\xi) d \xi\right)\right] d s \leq \mu(t)
$$


holds for t large enough. Then Eq. (1.1) has a positive solution $x(t)$ with the property $\left|\frac{x^{\prime}(t)}{x(t)}\right| \leq$ $\mu^{\frac{1}{\alpha}}(t)$.

Proof Let $\mu(t)$ be given such that the conditions of the theorem hold. We show that the conditions of Theorem 3.1 are satisfied with $\beta(t)=-\mu(t)$ and $\gamma(t)=\mu(t)$ for $t$ large enough.

Let $v(t)$ be a continuous function such that $|v(t)| \leq \mu(t)$. It follows from (3.5) that

$$
\begin{aligned}
|S v(t)| & =\left.\frac{1}{r(t)}\left|\alpha \int_{t}^{\infty} r(s)\right| v(s)\right|^{1+\frac{1}{\alpha}} d s+\int_{t}^{\infty}\left[\sum_{i=1}^{n} p_{i}(s) \exp \left(\alpha \int_{s}^{s+\tau_{i}(s)} v(\xi)^{\left(\frac{1}{\alpha}\right)^{*}} d \xi\right)\right] d s \mid \\
& \leq \frac{1}{r(t)}\left[\alpha \int_{t}^{\infty} r(s) \mu^{1+\frac{1}{\alpha}}(s) d s+\int_{t}^{\infty} \sum_{i=1}^{n}\left|p_{i}(s)\right| \exp \left(\alpha \int_{s}^{s+\tau_{i}(s)} \mu^{\left(\frac{1}{\alpha}\right)^{n}}(\xi) d \xi\right) d s\right] \\
& \leq \mu(t) .
\end{aligned}
$$

Therefore, by Theorem 3.1, Lemma 2.1 and Lemma 2.2, Eq. (1.1) has a positive solution, and the proof is complete.

Next, we consider neutral differential equations of the form

$$
\left[r(t)(y(t)-P(t) y(t-\tau))^{\prime}\right]^{\prime}+\sum_{i=1}^{n} p_{i}(t) f(y(t+\sigma))=0, \quad t \geq t_{0} .
$$

We assume that:

(i) $\tau>0, \sigma \geq 0$;

(ii) $r, P, p_{i} \in C\left(\left[t_{0}, \infty\right),(0, \infty)\right), i=1,2, \ldots, n$;

(iii) $f$ is nondecreasing continuous function and $x f(x)>0, x \neq 0$.

The following fixed point theorem will be used to prove the main results.

Lemma 3.1 (Schauder's fixed point theorem) Let $\Omega$ be a closed, convex and nonempty subset of a Banach space X. Let $T: \Omega \rightarrow \Omega$ be a continuous mapping such that $T \Omega$ is a relatively compact subset of $X$. Then $T$ has at least one fixed point in $\Omega$. That is, there exists an $x \in \Omega$ such that $T x=x$.

Theorem 3.3 Suppose that

$$
\int_{t_{0}}^{\infty} \sum_{i=1}^{n} p_{i}(t) d t=\infty
$$

and there exist $\zeta \geq 0,0<k_{1} \leq k_{2}$ such that

$$
\begin{aligned}
& \frac{k_{2}}{k_{1}} \exp \left[\left(k_{1}-k_{2}\right) \int_{t_{0}-\zeta}^{t_{0}} \sum_{i=1}^{n} p_{i}(t) d t\right] \leq 1 \\
& \exp \left(-k_{2} \int_{t-\tau}^{t} \sum_{i=1}^{n} p_{i}(t) d t\right)+\exp \left(k_{2} \int_{t_{0}-\zeta}^{t-\tau} \sum_{i=1}^{n} p_{i}(s) d s\right) \int_{t}^{\infty} \frac{1}{r(s)} \int_{s}^{\infty} \sum_{i=1}^{n} p_{i}(\xi) \\
& \quad \times f\left(\exp \left(-k_{1} \int_{t_{0}-\zeta}^{\xi+\sigma} \sum_{i=1}^{n} p_{i}(z) d z\right)\right) d \xi d s
\end{aligned}
$$




$$
\begin{aligned}
\leq & P(t) \\
\leq & \exp \left(-k_{1} \int_{t-\tau}^{t} \sum_{i=1}^{n} p_{i}(t) d t\right)+\exp \left(k_{1} \int_{t_{0}-\zeta}^{t-\tau} \sum_{i=1}^{n} p_{i}(s) d s\right) \int_{t}^{\infty} \frac{1}{r(s)} \int_{s}^{\infty} \sum_{i=1}^{n} p_{i}(\xi) \\
& \times f\left(\exp \left(-k_{2} \int_{t_{0}-\zeta}^{\xi+\sigma} \sum_{i=1}^{n} p_{i}(z) d z\right)\right) d \xi d s, \quad t \geq t_{0} .
\end{aligned}
$$

Then Eq. (3.6) has a positive solution which tends to zero.

Proof First: Choose $\widetilde{T} \geq t_{0}+\tau$,

$$
u(t)=\exp \left(-k_{2} \int_{t_{0}-\zeta}^{t} \sum_{i=1}^{n} p_{i}(t) d t\right), \quad v(t)=\exp \left(-k_{1} \int_{t_{0}-\zeta}^{t} \sum_{i=1}^{n} p_{i}(t) d t\right), \quad t \geq t_{0} .
$$

Let $C\left(\left[t_{0}, \infty\right), R\right)$ be the set of all continuous functions with the norm

$$
\|y(t)\|=\sup _{t \geq t_{0}}|y(t)|<\infty .
$$

Then $C\left(\left[t_{0}, \infty\right), R\right)$ is a Banach space. We define a closed, bounded convex subset $\Omega$ of $C\left(\left[t_{0}, \infty\right), R\right)$ as follows:

$$
\Omega=\left\{y \mid y \in C\left(\left[t_{0}, \infty\right), R\right): u(t) \leq y(t) \leq v(t), t \geq t_{0}\right\} .
$$

Define the map T : $\Omega \rightarrow C\left(\left[t_{0}, \infty\right), R\right)$ :

$$
(\mathrm{T} y)(t)= \begin{cases}P(t) y(t-\tau)-\int_{t}^{\infty} \frac{1}{r(s)} \int_{s}^{\infty} \sum_{i=1}^{n} p_{i}(\xi) f(y(\xi+\sigma)) d \xi d s, & t \geq \widetilde{T}, \\ (\mathrm{~T} y)(\widetilde{T})+v(t)-v(\widetilde{T}), & t_{0} \leq t \leq \widetilde{T} .\end{cases}
$$

We can show that for any $y \in \Omega$, Ty $y \in \Omega$.

Second: We prove that $\mathrm{T}$ is continuous.

Third: We show that $\mathrm{T} \Omega$ is relatively compact.

The proof is similar to Theorem 2.1 of [2], we omitted it.

Corollary 3.1 Suppose that $k>0$, (3.7) holds and

$$
\begin{aligned}
P(t)= & \exp \left(-k \int_{t-\tau}^{t} \sum_{i=1}^{n} p_{i}(t) d t\right)+\exp \left(k \int_{t-\zeta}^{t-\tau} \sum_{i=1}^{n} p_{i}(s) d s\right) \int_{t}^{\infty} \frac{1}{r(s)} \int_{s}^{\infty} \sum_{i=1}^{n} p_{i}(\xi) \\
& \times f\left(\exp \left(-k \int_{t_{0}-\zeta}^{\xi+\sigma} \sum_{i=1}^{n} p_{i}(z) d z\right)\right) d \xi d s, \quad t \geq t_{0} .
\end{aligned}
$$

Then Eq. (3.6) has a solution

$$
y(t)=\exp \left(-k \int_{t_{0}}^{t} \sum_{i=1}^{n} p_{i}(t) d t\right), \quad t \geq t_{0} .
$$


Example 3.1 Consider the advanced differential equations

$$
\left(x^{\prime}(t)\right)^{\prime}+\sum_{i=1}^{n} p_{i}(t) x(2 t)=0, \quad t \geq 2
$$

where $p_{i} \in C\left(\left[t_{0}, \infty\right), R\right)$ and $\sum_{i=1}^{n}\left|p_{i}(t)\right|=\frac{1}{8 \sqrt{2} t^{2}}$. Choose $\mu(t)=\frac{1}{2 t}$,

$$
\int_{t}^{\infty}\left(\frac{1}{4 s^{2}}+\frac{1}{8 \sqrt{2} s^{2}} \exp \left(\frac{1}{2} \int_{s}^{2 s} \frac{1}{\xi} d \xi\right)\right) d s=\int_{t}^{\infty} \frac{3}{8 s^{2}} d s \leq \frac{3}{8 t} \leq \frac{1}{2 t}
$$

All the conditions of Theorem 3.2 are satisfied. Equation (3.9) has a positive solution and $\left|\frac{x^{\prime}(t)}{x(t)}\right| \leq \frac{1}{2 t}$. In fact, we can choose $\mu(t)=1 /(\eta t), \eta \in(4-2 \sqrt{2}, 4+2 \sqrt{2})$, Eq. (3.9) has a positive solution with $\left|\frac{x^{\prime}(t)}{x(t)}\right| \leq \mu(t)$, and the solution satisfies $x(2) \cdot 2^{1 / \eta} \cdot t^{-1 / \eta} \leq x(t) \leq x(2)$. $2^{-1 / \eta} \cdot t^{1 / \eta}$

\section{Competing interests}

The authors declare that they have no competing interest.

\section{Authors' contributions}

The authors declare that the study was realized in collaboration with the same responsibility. All authors read and approved the final manuscript.

\section{Acknowledgements}

The authors sincerely thank the anonymous referees for their valuable suggestions and comments which greatly helped improve this article. Supported by NSF of China (11071054), Natural Science Foundation of Hebei Province (A2011205012).

\section{Received: 6 November 2012 Accepted: 20 May 2013 Published: 5 June 2013}

\section{References}

1. Agarwal, RP, Grace, SR, O'Reagan, D: Oscillation Theory of Second Order Linear, Half-Linear, Superlinear and Sublinear Dynamic Equations. Kluwer Academic, Dordrecht (2002)

2. Culáková, I, Hanuštiaková, L, Olach, R: Existence for positive solutions of second-order neutral nonlinear differential equations. Appl. Math. Lett. 22, 1007-1010 (2009)

3. Džurina, J, Stavroulakis, IP: Oscillation criteria for second order delay differential equations. Appl. Math. Comput. 140, 445-453 (2003)

4. Došlý, O, Řehák, P: Half-Linear Differential Equations. North-Holland Math. Stud., vol. 202. Elsevier, Amsterdam (2005)

5. Feng, MX, Xie, DX: Multiple positive solutions of multi-point boundary value problem for second-order impulsive differential equations. J. Comput. Appl. Math. 223, 438-448 (2009)

6. Györi, I, Ladas, G: Oscillation Theory of Delay Differential Equations with Applications. Clarendon, Oxford (1991)

7. Kusano, T, Lalli, BS: On oscillation of half-linear functional differential equations with deviating arguments. Hiroshima Math. J. 24, 549-563 (1994)

8. Kusano, T, Naito, Y, Ogata, A: Strong oscillation and nonoscillation for quasi linear differential equations of second order. Differ. Equ. Dyn. Syst. 2, 1-10 (1994)

9. Kusano, T, Yoshida, N: Nonoscillation theorems for a class of quasilinear differential equations of second order. J. Math. Anal. Appl. 189, 115-127 (1995)

10. Kulenovic, MRS, Hadziomerspahic, S: Existence of nonoscillatory solution of second order linear neutral delay equation. J. Math. Anal. Appl. 228, 436-448 (1998)

11. Luo, Y, Wang, WB, Shen, JH: Existence of positive periodic solutions for two kinds of neutral functional differential equations. Appl. Math. Lett. 21, 581-587 (2008)

12. Li, HJ, Yeh, CC: Nonoscillation criteria for second-order half-linear functional differential equations. Appl. Math. Lett. 8, 63-70 (1995)

13. Lin, XN, Jiang, DQ: Multiple positive solutions of Dirichlet boundary value problems for second order impulsive differential equations. J. Math. Anal. Appl. 321, 501-514 (2006)

14. Ladas, G, Sficas, YG, Stavroulakis, IP: Nonoscillatory functional differential equations. Pac. J. Math. 115, 391-398 (1984)

15. Péics, H, Karsai, J: Existence of positive solutions of halflinear delay differential equations. J. Math. Anal. Appl. 323, 1201-1212 (2006)

16. Shen, JH, Stavroulakis, IP, Tang, XH: Hille type oscillation and nonoscillation criteria for neutral equations with positive and negative coefficients. Stud. Univ. Zilina Math. Ser. 14, 45-59 (2001)

17. Tian, Y, Ji, DH, Ge, WG: Existence and nonexistence results of impulsive first order problem with integral boundary condition. Nonlinear Anal. 71, 1250-1262 (2009) 
18. Yu, YH, Wang, HZ: Nonoscillatory solutions of second-order nonlinear neutral delay equations. J. Math. Anal. Appl. 311, 445-456 (2005)

19. Zhang, WP, Feng, W, Yan, JR, Song, JS: Existence of nonoscillatory solutions of first-order linear neutral delay differential equations. Comput. Math. Appl. 49, 1021-1027 (2005)

20. Zhou, Y: Existence for nonoscillatory solutions of second-order nonlinear differential equations. J. Math. Anal. Appl. $331,91-96$ (2007)

doi:10.1186/1687-1847-2013-158

Cite this article as: $\mathrm{Li}$ et al.: Existence of positive solutions of advanced differential equations. Advances in Difference Equations 2013 2013:158.

Submit your manuscript to a SpringerOpen ${ }^{\circ}$ journal and benefit from:

- Convenient online submission

- Rigorous peer review

- Immediate publication on acceptance

Open access: articles freely available online

- High visibility within the field

- Retaining the copyright to your article

Submit your next manuscript at $\boldsymbol{s p r i n g e r o p e n . c o m ~}$ 